\title{
Morfología del empleo en las industrias de servicios intensivos en conocimiento. El caso de la Ciudad Autónoma de Buenos Aires
}

Cómo citar este artículo / To reference this article / Comment citer cet article / Para citar este artigo:

Martínez, R. G.; Leone, J. G.; Rodriguez-Repeti, J. M. (2020). Morfología del empleo en las industrias de servicios intensivos en conocimiento. El caso de la Ciudad Autónoma de Buenos Aires. Revista Escuela de Administración de Negocios, Edición especial 2020, 71 - 92. DOI: https://doi.org/10.21158/01208160.n0.2020.2740

\section{Resumen}

La Ciudad Autónoma de Buenos Aires se caracteriza por una estructura productiva intensiva en servicios, tanto en su absorción de mano de obra como en su participación en el producto. Los servicios intensivos en conocimiento (SIC) explican, a su vez, una buena parte de estos, de modo que merecen un cuidadoso análisis del tipo de demanda laboral asociada. Buena parte de la discusión académica redunda en la importancia de la oferta de capital humano para el desarrollo de la industria del conocimiento, por eso, entendido su rol en la proliferación del sector SIC, el objetivo principal de este trabajo es comprender este mecanismo, pero de forma inversa; es decir, entender la morfología de la demanda de empleo generada con base tanto en la calificación de la mano de obra como en sus tareas realizadas. Además de los controles habituales, se incorpora la precisión de la actividad laboral realizada, entendida bajo un específico esquema de tareas. El análisis permite concluir,

\footnotetext{
${ }^{1}$ Licenciado en Economía e investigador del Instituto Interdisciplinario de Economía Política de Buenos Aires. Profesor de la Facultad de ciencias económicas de la Universidad de Buenos Aires. ORCID: https://orcid.org/0000-0003-1699-5973

${ }^{2}$ Magíster en Economía. Investigador y profesor de la facultad de ciencias económicas de la Universidad de Buenos Aires. ORCID: https://orcid.org/0000-0002-8916-9798

${ }^{3}$ Licenciado en Economía. Investigador en formación del Instituto Interdisciplinario de Economía Política de Buenos Aires. ORCID: https://orcid.org/0000-0002-7899-9200
} 
entre otras cosas, que, aun con remuneraciones muy por encima del promedio, el control por tareas muestra una notoria disparidad salarial y en términos de creación de empleo. Al mismo tiempo, sobresale el sector SIC en la demanda laboral de puestos con mayor complementariedad tecnológica —intensidad en tareas cognitivas no rutinarias - y de calificación profesional y técnica.

Palabras clave: servicios intensivos en conocimiento; industria del conocimiento; demanda laboral; capital humano; sector servicios; creación de empleo.

\title{
$\longrightarrow$ Morphology of the employment in knowledge-intensive service industries. The case of the Autonomous City of Buenos Aires
}

\begin{abstract}
The Autonomous City of Buenos Aires is marked by an intensive productive structure in services, both in its absorption of labor and in its participation in the product. A good part of these are explained by the KnowledgeIntensive Services (KIS), so they deserve a careful analysis of the type of demand for labor that is associated. Much of the academic discussion revolves around the importance of supplying human capital for the development of the knowledge industry; therefore, with a clear understanding of its role in the proliferation of the KIS sector, the primary objective of this paper is to understand this mechanism, but in reverse; that is, to understand the morphology of the demand for labor that has been generated, based on both the qualification of the labor force and the tasks that are performed. Besides the usual controls, it incorporates the precision of the work activity, understood under a specific scheme of tasks. This analysis leads to conclude that even with salaries well above the average, the control by tasks shows a notorious gap in salaries and in terms of job creation. At the same time, the KIS sector stands out in the labor demand for positions with greater technological complementarity -intensity in non-routine cognitive tasks- and of professional and technical qualification.
\end{abstract}

Keywords: knowledge-intensive services; knowledge industry; demand for labor; human capital; service sector; job creation. 


\section{Morfologia do emprego nas indústrias de serviços intensivos em conhecimento. $\mathrm{O}$ caso da Cidade Autônoma de Buenos Aires}

\section{Resumo}

A Cidade Autônoma de Buenos Aires se caracteriza por uma estrutura produtiva intensiva em serviços, tanto na

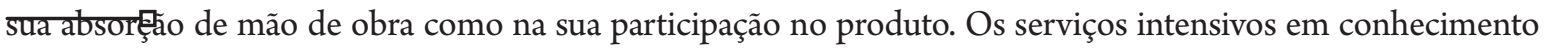
(SIC) explicam, por sua vez, boa parte destes, merecendo uma análise criteriosa do tipo de demanda de trabalho associada. Grande parte da discussão acadêmica redunda na importância da oferta de capital humano para o desenvolvimento da indústria do conhecimento, portanto, entendendo seu papel na proliferação do setor de SIC, o objetivo principal deste trabalho é entender esse mecanismo, mas inversamente; isto é, compreender a morfologia da demanda de trabalho gerada a partir da qualificação da mão de obra e das tarefas desempenhadas. Além dos controles usuais, incorpora-se a precisão da atividade de trabalho realizada, entendida sob um esquema específico de tarefas. A análise permite concluir, entre outras coisas, que, mesmo com salários bem acima da média, o controle por tarefas mostra uma notória disparidade salarial e de geração de empregos. Ao mesmo tempo, destaca-se o setor SIC na demanda de trabalho por cargos com maior complementaridade tecnológica —intensidade em tarefas cognitivas não rotineiras—e de qualificação profissional e técnica.

Palavras-chave: serviços intensivos em conhecimento; indústria do conhecimento; demanda de trabalho; capital humano; setor de serviços; criação de empregos.

\section{Morphologie de l'emploi du secteur des services hautement qualifiés. Le cas de la ville de Buenos Aires}

\section{Résumé}

La ville de Buenos Aires se caractérise par une structure productive à forte intensité de services utilisant une main d'oeuvre locale et qualifiée. Cet article s'interressera à ces services hautement qualifiés (SHQ) et analysera le type de main-d'œuvre associée. Si une grande partie de la discussion académique retient l'importance de la qualité des ressources humaines pour le développement de l'industrie du savoir et son rôle dans la croissance du secteur SHQ ce travail a pour objectif de comprendre ce mécanisme et la typolologie de la demande d'emploi générée selon les qualifications associées et les tâches exécutées par cette main d'oeuvre. L'analyse montre que, même avec des salaires bien supérieurs à la moyenne du secteur, le contrôle par tâches crée une importante disparité salariale et disparité en termes de création d'emplois. Dans le même temps, le secteur SHQ se démarque par une demande en main-d'œuvre pour des postes necessitant des connaissances et complémentarités technologiques, cognitives et techniques.

Mots-clés: services hautement qualifiés; industrie du savoir; demande de travail; ressources humaines; secteur des services; création d'emplois. 


\section{Introducción}

$\mathrm{T}$ radicionalmente, el sector de servicios ha sido asociado con empleos de menor productividad y escasos requerimientos educativos. En el último tiempo, el estudio del sector terciario obliga a un análisis segmentado en el que se destaca un subsector con características antagónicas a las mencionadas. Una parte de la industria, denominada precisamente por su intensidad en conocimiento, abarca actividades tan variadas como servicios contables y legales, arquitectura, audiovisuales, ingeniería, software, publicidad, investigación y desarrollo (I+D), o servicios de salud y educación.

La sofisticación del sector no exime a naciones en desarrollo de ocupar un rol en su provisión. Los últimos años mostraron las ventajas comparativas que la industria presenta en Argentina. Por una parte, su importancia en la dinámica exportable ubica al sector como el segundo mayor generador de divisas. Por otra, su intensidad en capital humano lo convierte en uno de los principales demandantes de mano de obra calificada, con remuneraciones muy por encima de las de otras industrias.

Sin embargo, la propia heterogeneidad de los sectores que lo componen hace que los servicios intensivos en conocimiento (SIC) repliquen este patrón a su demanda de trabajo. Esta no solo reacciona a los controles habituales — sexo, edad, nivel educativo-, sino que requiere, además, un análisis pormenorizado de las tareas que los empleados realizan. Al clásico esquema de habilidades, entendidas como una dotación de capacidades, se le añade un requerimiento específico de demanda. Es así como la industria del conocimiento articula un amplio abanico de actividades productivas que un primer análisis sectorial no logra captar.
Por una parte, la profesionalidad de la tarea se presenta como una dimensión de interés, entendiendo que las credenciales educativas individuales no necesariamente se equiparan a requerimientos homólogos en los organigramas empresariales. $\mathrm{Al}$ mismo tiempo, una óptica de intensidad en tareas con distinta capacidad de adecuación a los cambios tecnológicos tampoco resulta inocua en un sector que, por definición, sería compatible con estos.

Los servicios intensivos en conocimiento han potenciado una generación de trabajo con remuneraciones por encima de la media. Sin embargo, en el interior de la industria, la disparidad en el salario tanto mensual como horario resulta significativa, así como el tipo de absorción de empleo. La introducción de controles por tareas presenta resultados contundentes y de enormes amplitudes en el interior de estas. La funcionalidad y especificidad de la actividad laboral ostenta un potencial explicativo mucho más alto que el de la industria en sí. Si bien no debe obviarse que estas tareas se desarrollan en el marco de un sector que las demanda, el amplio abanico de tareas obliga a una mirada más exhaustiva que hasta el momento no se había incorporado en el interior del sector.

Buena parte de la discusión académica redunda en la importancia de la oferta de capital humano para el desarrollo de la industria del conocimiento. Entendido su rol en la proliferación del sector SIC, será objetivo principal de este trabajo comprender este mecanismo, pero de forma inversa; entender la morfología de la demanda de empleo generada con base tanto en la calificación de la mano de obra como en sus tareas realizadas. 
Este documento toma como universo de análisis La Ciudad Autónoma de Buenos Aires (CABA), destacada por un esquema productivo fuertemente ligado a la provisión de servicios ${ }^{4}$. En la actualidad, más del $90 \%$ de su producto bruto geográfico (PBG) se articula en este segmento, representando una muestra robusta de cara a la construcción de instrumentos útiles. Las distintas herramientas de análisis se enmarcan en el objetivo general, de manera que llega a este mediante metas particulares: a) comparar ingreso medio y generación de empleo SIC, resto de servicios y sector bienes; b) determinar su distribución geográfica; $\mathrm{c}$ ) identificar y categorizar los tipos de tarea que se realizan en el interior de cada sector; y d) observar el impacto de diferentes dotaciones individuales, en cuanto características de demanda de los puestos laborales.
La estructura del trabajo comienza por un marco teórico que recopila la bibliografía relevante para el empleo en el sector SIC. Luego, se presenta la metodología y las fuentes de datos utilizadas en el momento del tratamiento de la información. La siguiente sección analiza los principales resultados en torno al volumen y la remuneración media en el empleo dentro del sector en CABA, diferenciando por zonas geográficas, el tipo de tareas y la calificación de estas —requerimientos de los puestos-. En la última sección se examina el potencial explicativo de las características individuales de cara a desempeñarse tanto dentro de las industrias SIC como en tareas específicas en el interior de estas.

\section{Marco teórico}

$\mathrm{T}$ anto la globalización como el auge de las cadenas globales de valor han modificado el esquema de producción y la radicación del valor agregado. Un esquema donde el what you do es mucho más trascendente que el what you sell configura una estructura productiva en la que, difícilmente, un agente económico integre todo un proceso. En este contexto, el sector servicios presenta una particular dinámica bajo una creciente valuación de activos intangibles (OECD, 2013).

Históricamente, el sector terciario ha sido asociado a niveles de calificación técnica más baja con respecto al tradicional esquema industrial-fabril, como resultan las tareas de asistencia y cuidado de personas o servicios personales. Usualmente se ligaban a ocupaciones de baja productividad, menor innovación y de carácter no transable. Sin embargo, el auge de las industrias SIC puso a prueba este paradigma. En países desarrollados con una matriz tecnológica esperablemente avanzada, desde hace tiempo, el sector servicios supera en participación al de bienes tanto en producto como en generación de empleo (López, Niembro y Ramos, 2017).

Mientras, inicialmente, su provisión se realizaba «fronteras adentro», las industrias SIC impulsaron un marcado proceso de «transabilidad» a nivel internacional. Este tipo de servicios engrosaron el flujo de comercio, de modo que llegaron a explicar el $20 \%$ del total de las exportaciones (Escaith, 2008). Una dinámica similar surgía en la creación de valor agregado y la injerencia en los esquemas globales de transnacionalización. Servicios tales como empresariales, transporte y logística representaban el $50 \%$ de la creación de valor

\footnotetext{
${ }^{4}$ CABA contiene el PBG per cápita más elevado del país. Para el 2004, con base en el cálculo de las cuentas nacionales, este fluctuaba en alrededor de ARS 28500 anuales — pesos corrientes 2004_, casi el triple de aquellas jurisdicciones importantes con un PBG elevado como Buenos Aires, Córdoba y Santa Fe.

${ }^{5} \mathrm{Si}$ bien estas resultan las cifras conocidas, es esperable que este sea el piso y no el techo de las exportaciones, fruto de las dificultades de contabilización y valuación.
} 
agregado, con una fuerte penetración en el esquema global de transnacionalización: más del $70 \%$ de las importaciones del segmento eran servicios intermedios. Las regiones más desarrolladas resultan ser las de mayor participación para el sector servicios, alcanzando casi dos terceras partes del PBI (OECD, $2013)^{6}$.

Las industrias SIC se encuentran ligadas a la dotación de capital humano del país en el que ofrecen sus servicios (Miles et al., 1995). No obstante, naciones que no ocupan un rol geopolítico central y no resultan pioneras en la dotación de capital humano pueden ocupar un lugar destacado en este tipo de industrias. Sin embargo, no son pocos los países en cursos de desarrollo con mucho para aportar en el segmento. Latinoamérica presenta ejemplos de notorios avances en el sector, con potencial para transformarse en un motor de crecimiento a pesar de encontrarse aún muy lejos de su tope (López y Ramos, 2013).

Al mismo tiempo, Meyer (2007) destaca el rol de las ventajas comparativas en la dotación de capital humano, proponiendo una metodología alternativa para el caso de India ${ }^{7}$. Concluye que incluso países con producto bruto interno $(\mathrm{PBI})$ per cápita bajo $\mathrm{y}$, por ende, salarios menores, pero con relativa acumulación de capital humano y afinidad en el idioma - proxy de los costos de transacción-, pueden beneficiarse de este tipo de industrias.

La evidencia empírica muestra que Argentina puede incluirse en este grupo, con un protagonismo diversificado en varios de sus componentes, por ejemplo, para el 2013 según la United Nations Conference on Trade and Development (UNCTAD),
Argentina aparecía segunda en la región — detrás de Costa Rica - en cuanto a servicios de informática, aunque muy lejos del podio emergente ocupado por India, país que presentaba exportaciones 30 veces superiores. Para el caso de servicios personales, culturales y recreativos, los cuales incluyen salud, educación y audiovisuales, Argentina se encontraba primera en la región y con una distancia mucho más corta respecto a Hungría, nación que encabeza las economías emergentes y triplicaba nuestras exportaciones.

Sin embargo, López y Ramos (2013), con base en datos de A. T. Kearney, analizan los principales determinantes para la aparición de este tipo de industrias en América Latina. Además del entorno de negocios, la capacidad y disponibilidad de la fuerza laboral y el atractivo financiero, la variable educación presenta las mayores deficiencias para casi toda la región. Por su parte, no debe omitirse el déficit de vocaciones en ciertas áreas académicas — como, por ejemplo, las ingenieriles en general, o la computación en particular-, hecho generalizado en Occidente y particularmente relevante en América Latina.

Adicionalmente, Guerrieri y Meliciani (2009) concluyen que el sector, además de su dependencia frente a la inversión en investigación y desarrollo (I+D) y capital humano, verifican también la presentación de un esquema de economías de aglomeración. Regiones lindantes a ciudades capitales denotan una mayor absorción de empleo para este tipo de industrias. El clivaje regional cobra una especial importancia ligada a un mayor desarrollo en centros urbanos y grandes aglomerados que justifican un estudio específico.

\footnotetext{
${ }^{6}$ Mientras en términos de exportaciones su importancia disminuye — representan un cuarto de las exportaciones en bienes-, al contabilizar el valor agregado su peso se amplía fuertemente.

${ }^{7}$ La India resulta uno de los países más destacados en los sectores SIC. Con una ponderación del $16 \%$ de sus exportaciones entre el 2000 y el 2003, relacionada con servicios informáticos, el offshoring explicaba esta característica no captada por los modelos tradicionales pensados para naciones centrales y con elevadas tasas de ahorro.
} 
Este documento se propone un análisis de intersección entre las industrias SIC y su mercado laboral asociado. Usualmente suele afiliarse una característica laboral con el rubro en el que está empleando al individuo. Sin embargo, incluso en el interior de un mismo sector, la tarea que efectivamente realiza puede tener considerables diferencias. $\mathrm{El}$ «enfoque de tareas» representa una buena aproximación a las habilidades del trabajador, si se entiende una correspondencia entre ambas.

Autor et al. (2003) y Acemoglu y Autor (2011) utilizan esta metodología con el fin de evaluar los potenciales riesgos de automatización, no en función de los atributos individuales, sino de la tarea desempeñada. El conjunto de tareas en cuatro principales vertientes que combinan dos amplias categorías son: a) rutinario, b) no rutinario, c) manual, d) cognitivo. En cuanto a la primera agrupación — puntos a y b - la diferencia radica en si la tarea es un conjunto repetido de acciones invariantes o no. En caso de ser así y tratarse de una repetición metódica en torno a un procedimiento constante, estas claramente pueden ser imitadas por una máquina o un programa informático. Por su parte, en cuanto a la segunda distinción - puntos cy d-, el uso de la fuerza física separa ambos grupos de tareas para dar lugar a las distintas combinaciones (Frey y Osborne, 2013).

Dentro del sector servicios se destacan aquellos trabajos intensivos en tareas manuales no rutinarias asociadas a trabajos de baja calificación y retorno económico. Ya el antiguo Dictionary of Occupational Tasks (DOT) definía a estas tareas en torno a la capacidad para mover manos y pies - entre otros miembros - de acuerdo con estímulos visuales. Para Estados Unidos, Autor y Handel (2013) mostraban un proceso de polarización en el empleo en el que el aumento en la participación de la cola más baja de la distribución se explicaba por el crecimiento de servicios tales como limpieza, cuidado personal, peluquería, venta ambulante, etc.

Aquellos trabajos intensivos en tareas rutinarias, bien sea cognitivas o bien manuales, enumeran los mayores desafíos en el escenario laboral, con un riesgo de automatización - y por ende de caída en la absorción de empleo- muy elevado. En un informe de dividendos digitales, el Banco Mundial (2016) estima que el $50 \%$ del empleo en América Latina podría no existir en el futuro. Si bien desde la primera revolución industrial este fenómeno se vio ligado a los empleos manuales, la revolución tecnológica ha demostrado que las máquinas también pueden, cómodamente, sustituir tareas cognitivas manuales. De acuerdo con Bresnahan (1999), durante las últimas tres décadas las computadoras habían sustituido ocupaciones asociadas con el cálculo, la coordinación de actividades y la comunicación, cajeros de bancos, operadores telefónicos y otros operarios de tareas repetitivas de procesamiento de información.

El escenario descripto enuncia una polarización definida tanto en las tareas desarrolladas como a nivel salarial. En este sentido, son los empleos de calificación media los que en mayor medida disminuyen su participación, mientras se observa un crecimiento en las calificaciones de esquina, es decir, las calificaciones altas y bajas (Autor y Handel, 2013). En la misma línea, se observaron aumentos en la desigualdad salarial con «premios» a la educación ligados a la estructura productiva. Para el caso de EE. UU., mientras las brechas salariales por nivel educativo se acortan desde 1915 a 1980 (Goldin y Katz, 2007), tanto las brechas educativas como la desigualdad salarial han exhibido un fuerte aumento desde 1980 en adelante en muchos países (Atkinson, 2008; Goldin y Katz, 2007; Murphy, Riddell y Romer, 1998).

Esto puede derivarse de una aceleración en la complementariedad entre capital y mano de obra calificada, producto de la adopción de computadoras y tecnología informática (Katz y Krueger, 1998; Bresnahan, Brynjolfsson y Hitt, 2002). Por el contrario, Picketty y Saez (2014) se distancian de la «carrera» entre educación —oferta calificaday tecnología — demanda calificada—, de modo que ponen el foco en las instituciones laborales y la política impositiva como herramienta orientada a contrarrestar la desigualdad laboral. 
Dauth et al. (2017), para el caso alemán ${ }^{8}$, verifican también un alza en los empleos calificados asociados a posiciones científicas y de management, mientras los de baja calificación, —y, sobre todo, los medios ligados a utilización de maquinaria — se vieron especialmente perjudicados. En particular, si bien este estudio no observa una destrucción de empleos existentes, sí evidencia una menor creación de empleos ligados a la industria manufacturera para quienes se incorporan al mercado laboral.

En la misma línea, Acemoglu y Restrepo (2017a; 2017b; 2018) plantean un equilibrio en el que colisionan dos fuerzas: el efecto desplazamiento y la productividad. El primero, lógicamente, refiere a la capacidad de sustitución de trabajadores, mientras que el segundo se explica por la reducción de costos y su correspondiente incremento en la producción y la demanda de empleo en las industrias en que se encuentra instalado. De este modo, los trabajadores pueden ser absorbidos por diferentes industrias, especializándose en nuevas tareas complementarias a la robótica.

Por último, Apella y Zunino (2017) muestran para Argentina y Uruguay que el incremento de la proporción de trabajadores calificados deriva en una mayor probabilidad de desempeñar tareas cognitivas no rutinarias - complementarias con la innovación tecnológica y la robótica- en detrimento de aquellas de carácter manual. Concluyen que el viraje hacia empleos intensivos en tareas cognitivas no rutinarias en Argentina fue producto, por una parte, de un cambio en las ocupaciones en el interior de una misma rama de actividad, en la que el rol del nivel educativo suele ser determinante?

\footnotetext{
${ }^{8}$ Alemania presenta una elevada participación tanto en robótica como en empleo manufacturero.

9 Se verifican también cambios en el interior de las ocupaciones y, por ende, en las tareas requeridas dentro de una misma ocupación. Por el contrario, destacan que la contribución del cambio estructural — migración del sector agropecuario al terciario, por ejemplo— ha sido prácticamente nula para las últimas dos décadas del caso argentino.
} 


\section{Metodología}

$\mathrm{C}$ omo insumo principal se utiliza la información estadística relevada por la Dirección General de Estadísticas y Censos de la Ciudad de Buenos Aires (DGEyC-GBA), institución que recolecta los microdatos socioeconómicos tanto a nivel individual como por hogar. En este caso, se utilizan las bases usuarias de las encuestas anuales de hogares (EAH), publicadas por el departamento de estadísticas de la Ciudad Autónoma de Buenos Aires para los años 2013, 2015 y 2018.

De cara a un análisis de heterogeneidades en el interior de una macrociudad, se procede a agrupar las comunas en sectores geográficos más amplios -Norte, Sur, Oeste y Centro-Este- Además de la proximidad, estas regiones comparten similares atributos socioeconómicos en su población. La composición por zonas se relacionó, en primer término, por la vecindad, y luego, adecuada por la extensión territorial de cada área, alcanzó alrededor de unos $50 \mathrm{~km} 2$ cada una (Profac B, 2018) ${ }^{10}$.
Posteriormente, se determinan diferentes categorías por rama de actividad según la clasificación de las encuestas para actividades sociodemográficas Caes-Mercosur 1.0—, versión para la Argentina ${ }^{11}$, diferenciando las ocupaciones de servicios intensivas en conocimiento y sus respectivas ramas (véase la Tabla 1). Esta diferenciación surge a partir del ordenamiento parte del código de la Encuesta Trimestral de Ocupación e Ingresos (ETOI) ${ }^{12}$. Además, se tipifican el resto de los servicios y la producción de bienes. Para cada una de ellas se analiza el tamaño del sector — cantidad de ocupados- y su respectivo ingreso medio, considerando el promedio ponderado de la remuneración a la ocupación principal. En caso de no encontrarse disponible el dato, se utiliza el ingreso total del encuestado ${ }^{13}$.

\footnotetext{
${ }^{10}$ La población radicada en cada una de las zonas seleccionadas fue la siguiente — sobre datos censales del año 2010—: la CentroEste concentró la mayor cantidad de vecinos, con alrededor de un millón de personas — 32,2 \% de la población total de CABA-, seguida por la Norte - 28,2 \% - Sur - 21,3\%—y Oeste - 17,9\%—. La zona Norte se caracterizó por contener a una población envejecida y con elevados niveles educativos, lo que redundó en un ingreso medio superior en el resto de las zonas; en contraste, la zona Sur mostró una población joven, con alta tasa de natalidad, aunque el ingreso medio fue el menor de la ciudad —inclusive padecieron las mayores carencias sociales-. Cabe destacar la brecha entre los ingresos medios de ambas zonas, la cual rondó el 35 \% - 2015 - . Por su parte, la zona Centro-Este presentó indicadores más asociados a la zona Norte y la Oeste a la Sur.

${ }^{11}$ El Clasificador Nacional de Ocupaciones se encuentra descripto en las bases de datos como la variable t37_cod_2.

${ }^{12}$ La ETOI es un operativo de la DGEyC-GBA que permite la producción sistemática y permanente de indicadores laborales y de ingresos de los hogares y los habitantes de la ciudad.

${ }^{13}$ Para el 2013 no se disponía del ingreso de la ocupación principal, por tanto, el ingreso medio para cada categoría se determinó como el promedio ponderado del ingreso total percibido por la persona, lo que impedía realiza una comparación lineal con los años posteriores. Tanto para la cantidad de ocupados como para el ingreso medio se utilizaron los factores de expansión presentes en las respectivas encuestas anuales de hogares.
} 
Tabla 1. Clasificación de los servicios intensivos en conocimiento

\begin{tabular}{|c|c|}
\hline Servicios empresariales & \\
\hline 6900: Actividades jurídicas y de contabilidad. & \\
\hline 8200: Actividades administrativas de oficinas y otras actividades auxiliares de las & empresas. \\
\hline 7800: Actividades relacionadas con el suministro de empleo. & \\
\hline 6300: Actividades de servicios de información. & \\
\hline 7000: Actividades de servicios de consultoría en gestión empresarial. & \\
\hline 9401: Actividades de organizaciones empresariales, profesionales y de empleador & \\
\hline Servicios de salud & \\
\hline 8600: Actividades de atención a la salud humana. & \\
\hline 8700: Asistencia social relacionada con la atención a la salud. & \\
\hline Servicios de educación & \\
\hline 8501: Enseñanza inicial y primaria, secundaria y superior. & \\
\hline 8502: Otros tipos de enseñanza n. c. p., y servicios de apoyo. & \\
\hline Servicios financieros & \\
\hline $\begin{array}{l}\text { 6400: Intermediación financiera y otros servicios financieros, excepto los de segu } \\
\text { jubilaciones y pensiones. }\end{array}$ & ros y administración de fondos de \\
\hline 6500: Seguros, reaseguros y fondos de pensiones, excepto los planes de seguridad & social de afiliación obligatoria. \\
\hline 6600: Actividades auxiliares a los servicios financieros y a los seguros. & \\
\hline Servicios informáticos & \\
\hline 6200: Actividades de programación y consultoría informáticas y otras actividades & conexas. \\
\hline 9501: Reparación de equipos informáticos. & \\
\hline Industrias creativas & \\
\hline 5900: Actividades cinematográficas, producción de videos y programas de televis & ón, grabación sonora y edición de música. \\
\hline 7100: Servicios de arquitectura e ingeniería; ensayos y análisis técnicos. & \\
\hline 7301: Actividades publicitarias. & \\
\hline 8102: Servicios de paisajismo y jardinería. & \\
\hline 9000: Actividades artísticas y de espectáculos. & \\
\hline Otros servicios intensivos en conocimiento & \\
\hline 3300: Mantenimiento, reparación e instalación de máquinas y equipos. & \\
\hline 6100: Telecomunicaciones. & \\
\hline 7200: Investigación y desarrollo. & \\
\hline 7302: Investigación de mercados y encuestas de opinión pública. & \\
\hline 7400: Otras actividades profesionales, científicas y técnicas. & \\
\hline 8000: Actividades de investigación y seguridad. & \\
\hline 9100: Actividades de bibliotecas, archivos, museos y otras actividades culturales. & \\
\hline 9302: Actividades de entretenimiento n. c. p. & \\
\hline 9502: Reparación de equipos de comunicación. & \\
\hline
\end{tabular}

Fuente. Elaboración propia con base en López y Ramos, 2013. 
Posteriormente, se realiza un análisis escindido por tareas, a diferencia del clásico esquema de habilidades. De este modo, teniendo en cuenta la clasificación nacional de ocupaciones (CNO2001) y la Clasificación Internacional Uniforme de Ocupaciones, versión 2008 (CIUO-08) ${ }^{14}$, se determina qué actividad pertenecía a cada rama productiva y se clasifican las ocupaciones de acuerdo con la intensidad de las tareas que la componen ${ }^{15}$.

Al mismo tiempo, se determinan por rama productiva cuatro típicas clasificaciones de ocupaciones de acuerdo con la intensidad de las tareas desarrolladas: manuales rutinarias, las cuales son tareas asociadas con la destreza en los dedos y la manipulación de objetos pequeños realizadas por trabajadores con media calificación, aunque altamente codificables y reemplazables por procesos de automatización; manuales no rutinarias, que conllevan poca calificación pero resultan poco codificables, de manera que influyen en su realización factores como, por ejemplo, los culturales, que dificultan su automatización; cognitivo rutinarias, realizadas por trabajadores de calificación media, se pueden computarizar — dada su alta repetición - y son ejemplo de ellas las tareas desempeñadas por el personal administrativo o el personal de ventas, entre otros; y cognitivas no rutinarias, que son aquellas tareas realizadas por personal altamente calificado $\mathrm{y}$ requieren pensamiento abstracto, creatividad, capacidad de resolver problemas de manera crítica y habilidades comunicacionales —estas últimas complementan la informática y la tecnología- Esta clasificación se formula con base en la información expuesta por la $\mathrm{O}^{*} \mathrm{NET}$ (Occupational Information Network), lo que permite clasificar el contenido de tareas de las ocupaciones para el caso de Estados Unidos $^{16}$.

De cara a las condiciones de empleabilidad del sector, se evalúa la importancia del tipo de tarea en un análisis multifacético, a diferencia de una simple delimitación del entorno sectorial. Se plantean así cuatro variables explicadas de tipo dicotómica, enmarcadas en un modelo de regresión logística — logit_, pues es uno de los métodos más sencillos y confiables -en términos estadísticos - para el procesamiento de la información con la que se trabaja.

En primer lugar, se analiza la probabilidad de alcanzar un empleo SIC acotando la muestra en aquellas personas con edad de trabajar - 18 a 65 años-, y que no se declaren inactivas. En segunda instancia, mediante el código SICCOGN, se toma como variable explicada la probabilidad de desempeñarse en una industria SIC, desarrollando una tarea que haya sido calificada como cognitiva no rutinaria. A través de la denominación SICs/ed, alcanzan el valor 1 todos los rubros de la industria SIC, a excepción del sector educativo, de cara a analizar la inserción femenina en el resto de la industria - las mujeres presentan una elevada participación en las labores ligadas a la enseñanza-. Por último, por medio del código SICPROF se analiza el arribo a aquellos rubros de la industria SIC que presentan una calificación ocupacional profesional - de acuerdo con el clasificador nacional de ocupaciones a cinco dígitos-.

\footnotetext{
${ }^{14}$ El Clasificador Internacional Uniforme de Ocupaciones organiza los empleos en una serie de grupos definidos en función de las tareas que comporta cada empleo.

${ }^{15}$ Descriptas en las bases usuarias con el código «t41_cod_2».

${ }^{16}$ Se agradece el valioso aporte de Ignacio Apella en torno a la clasificación de tareas. Al mismo tiempo, se advierten potenciales sesgos al asumir que las características de las ocupaciones argentinas son idénticas a las norteamericanas.
} 


\section{Resultados}

\subsection{El sector Servicios Intensivos en Conocimiento en CABA}

La Ciudad Autónoma de Buenos Aires se caracteriza por una estructura productiva intensiva en servicios: su peso en el producto bruto geográfico rondó el $77 \%$ durante las últimas tres décadas. Esto denota una tendencia sistemáticamente creciente, ya que se situaba para inicios de la década de los noventa en el orden del $73 \%$, mientras que en el 2016 este valor alcanzaba el $80 \%$. Por su parte, los datos de empleo en servicios sugieren una importancia aún más alta: nueve de cada diez ocupaciones se enmarcaban dentro del sector.

Con un crecimiento acumulado del $60 \%$ entre el 2004 y el 2016 en el PBG de CABA — 4 \% anual—, las ramas SIC explicaron buena parte de esta suba. La importante contribución al crecimiento de la ciudad ya representaba un hecho estilizado, interrumpido exclusivamente por el periodo recesivo del 2001. Aun agrupando muchas menos industrias que el sector

Figura 1. Tamaño del sector e ingreso medio servicios convencionales y bienes, para la última fase cíclica logró un poder de tracción superior al resto de la economía.

Con respecto al empleo porteño, los rubros SIC se desagregan en cuatro subgrupos de acuerdo con la cantidad de empleo generada y la remuneración media otorgada (véase la Tabla 2). En cuanto a los de alta demanda, se destaca una participación similar en servicios empresarios, de salud y educación. Sin embargo, el diferencial de ingresos es notorio, dado que pertenece el primero, principalmente, a actividades de gestión privada. En cambio, los dos últimos plantean un fuerte componente de gestión estatal, con remuneraciones por fuera de valores de mercado, y en este caso por debajo de la media. Así, por ejemplo, en educación el ingreso promedio es inferior a aquellos informados por ocupaciones relacionadas con la producción de bienes y servicios No SIC —ubicados en la parte más baja de la distribución, con una influencia del sector comercio muy significativa一.

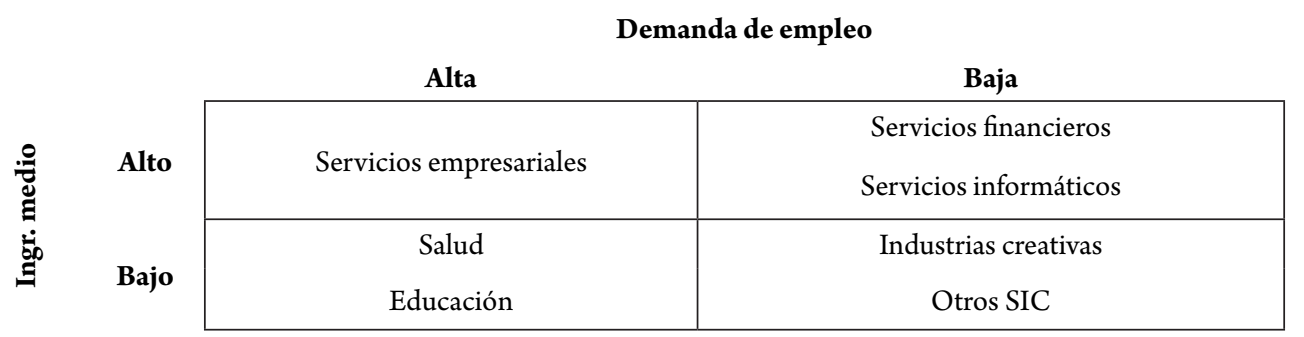

Fuente. Elaboración propia con base en datos de EAH de la DGEyC de CABA.

La combinación tamaño del sector e ingreso medio se ilustra en la figura 2. Dentro de las ramas SIC, las ocupaciones en servicios empresariales comprenden un tamaño apreciable y se distinguen por su ingreso medio relativamente elevado. Por su parte, los financieros e informáticos presentaron un ingreso medio mayor, aunque con una planta mucho más reducida. Por el contrario, sectores asociados a salud y educación mostraron un ingreso medio más bajo, así como la mayor absorción de empleados. 
Figura 2. Ingreso medio mensual y cantidad de empleados por rama de industria SIC

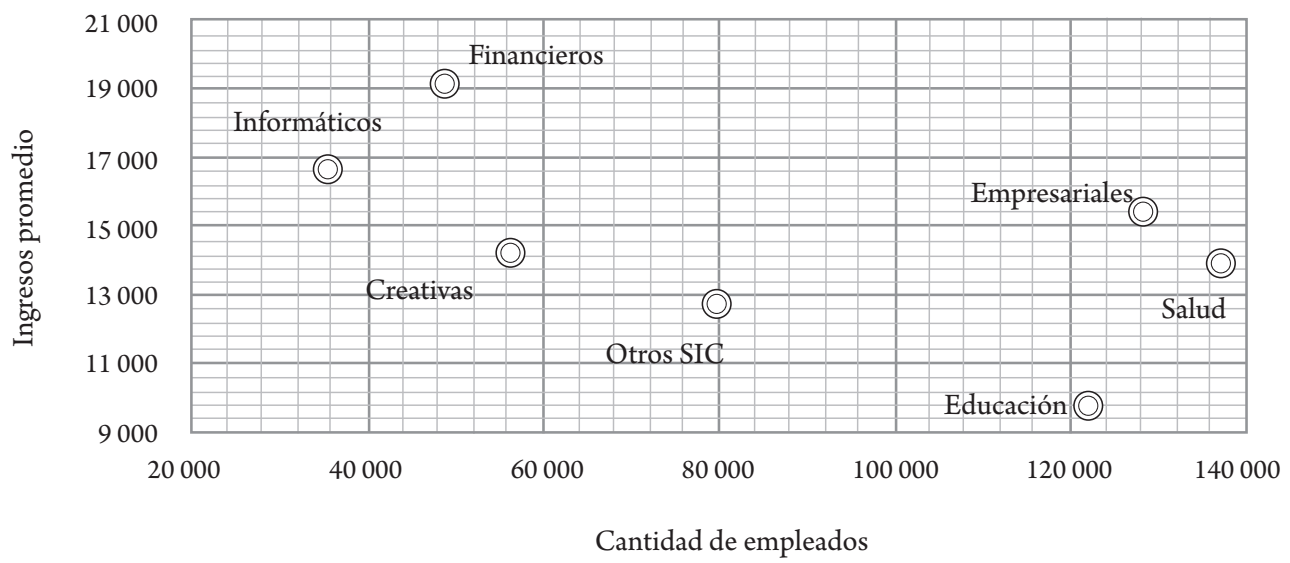

Fuente. Elaboración propia con base en datos de EAH de la DGEyC de CABA.

El perfil de las industrias SIC dista mucho de ser uniforme, incluso en el interior de una ciudad. Con distintas combinaciones de ingreso medio y nivel de empleo, no parece encontrarse una correlación lineal en la industria. Sin embargo, la participación por rubro parece mostrar un lógico patrón de comportamiento en torno a las áreas geográficas de CABA: aquellas de nivel socioeconómico más elevado son las que mayor participación presentan en los sectores de ingresos medios altos.

Figura 3. Distribución geográfica del empleo en 2015

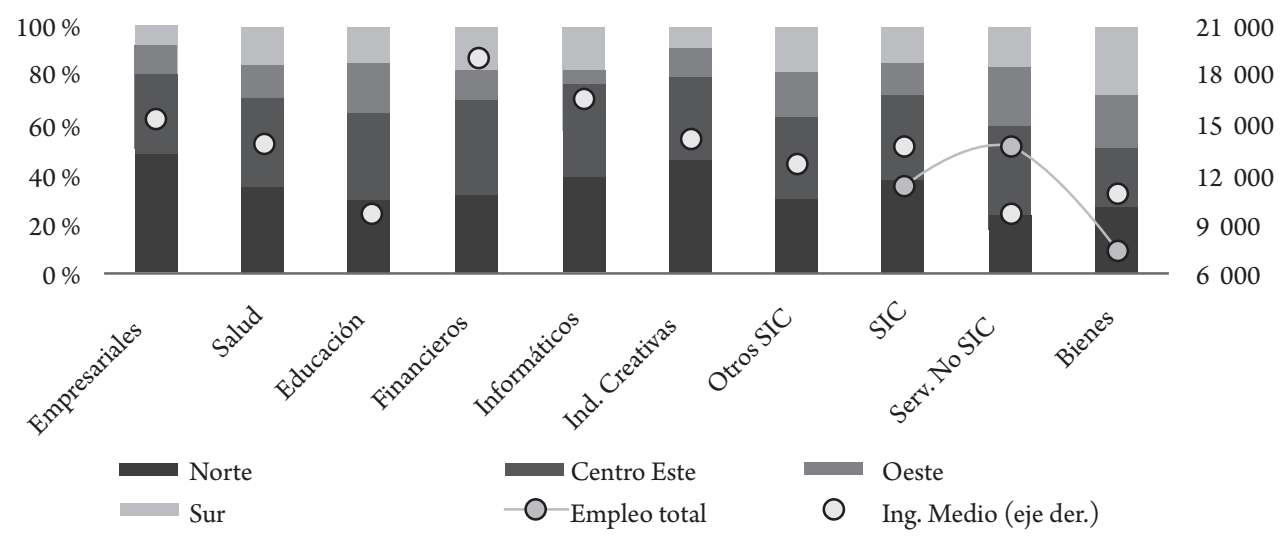

Fuente. Elaboración propia con en base a datos de EAH de la DGEyC de CABA.

La figura 3 muestra la importancia de las zonas Norte y Centro-Este en la localización de los empleados de las industrias SIC. Considerando la relevancia de la dotación de conocimiento en este tipo de industrias, no es llamativo que sean aquellas zonas con mayores complejidades en materia educativa las que encuentren empleo en industrias con menor demanda de empleo calificado. De acuerdo con Martínez, Lo Cascio y Leone (2018), allí en donde se señalan las carencias educativas, principalmente de la zona Sur, se asocian a desarrollos sectoriales más limitados en industrias de mayores requerimientos de calificaciones. 


\subsection{Enfoque por tareas}

4.2.1 Industrias SIC y riesgos de automatización.

Con una participación determinante del sector servicios, $\mathrm{CABA}$ presenta dos grandes agrupamientos con características distantes en torno a su demanda de trabajo. El sector intensivo en conocimiento nuclea casi el $65 \%$ de su empleo ligado a tareas cognitivas no rutinarias, mientras que para los servicios convencionales menos del $20 \%$ pueden enmarcarse en esta categoría. Más aún, no solo se destaca la menor complementariedad con tecnología, sino también la mayor participación de empleos intensivos en tareas rutinarias. En efecto, un tercio de los empleos del sector servicios No SIC — con una absorción de más de la mitad del empleo porteño- exhibe un fuerte riesgo de automatización en sus tareas (véase la Figura 4).

Figura 4. Tipo de tareas por sector y subsector de ocupación

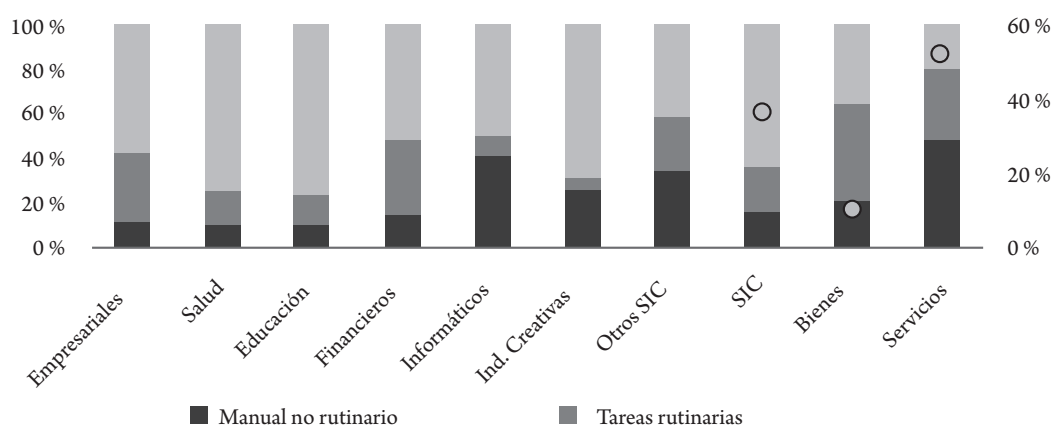

Fuente. Elaboración propia en base a datos de EAH de la DGEyC de CABA y O*NET.

Estos resultados son coincidentes con los hallazgos para países desarrollados, en los que la intensidad en tareas manuales no solo alcanza la producción de bienes y actividades operativas ligadas a la utilización de maquinaria, sino también en servicios tales como el comercio minorista o una buena parte del trabajo de oficina. Sin embargo, destacan al sector servicios a nivel agregado por su intensidad en tareas manuales - transporte, por ejemplo-, de manera que presenta una demanda de empleo de calificación baja (Autor y Dorn, 2013). Para el caso de CABA, casi la mitad de los trabajos generados por el sector servicios No SIC se relacionan con tareas manuales no rutinarias.
4.2.2 SIC y demanda de trabajo calificado.

Tal como su nombre lo indica, no es extraño observar la fuerte presencia de tareas profesionales en el sector servicios intensivos en conocimiento, en el cual las tareas operativas - comúnmente asociadas a labores intensivas en tareas manualesy no calificadas apenas alcanzan el $30 \%$ de la demanda total de empleo. Por el contrario, tanto la demanda de empleo ligada a la producción de bienes como los servicios convencionales presentan una segmentación prácticamente idéntica, destacándose exclusivamente una mayor participación de la población con menor instrucción para este último. De nuevo, el sector servicios aflora como el principal demandante de aquellos trabajadores con nula instrucción. 
Figura 5. Calificación ocupacional por sector y subsector

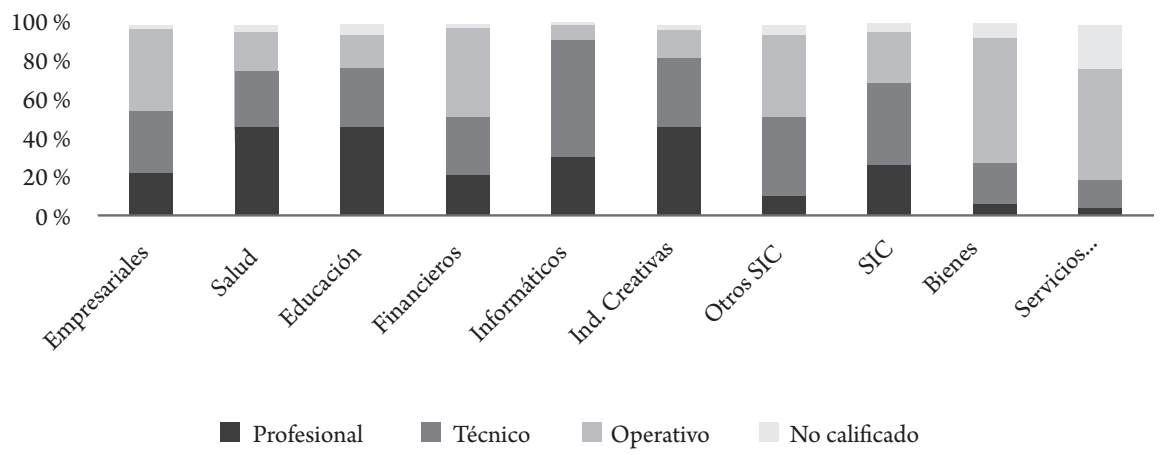

Fuente. Elaboración propia con base en datos de EAH de la DGEyC de CABA.

Sin embargo, a pesar de no ser llamativa la composición del empleo para el sector SIC, sí se destaca por ser el único en presentar una demanda calificada significativa. Tanto para el resto de los servicios como para el de bienes, menos del $5 \%$ de sus trabajadores presentan una calificación profesional. En el interior de este, salud e industrias culturales - en mayor medida- y servicios informáticos se ubican por encima de la participación media, de modo que son estos dos últimos los de menor peso en el total (véase Figura 5).

Para este último caso, Nyahoho (2010) destacaba especialmente el potencial explicativo de la dotación de capital humano en la exportación de estos sectores. Sin embargo, también debe considerarse al empleo técnico como calificación alta, en el cual los servicios informáticos o educativos representan la mayor parte del total. En este caso, el peso de educación terciaria no universitaria - tecnicaturas - resulta significativo y es otra arista del empleo calificado que no posee las máximas credenciales educativas.

\subsection{Ingreso horario por sector y ocupación}

Si bien el ingreso mensual nos brinda un claro panorama de la remuneración por sector, a efectos analíticos se utiliza el salario horario. De este modo, se cuenta con una herramienta homogénea que permite eliminar los efectos del empleo a tiempo parcial (véase la Figura 6). Por su parte, nos permite analizar la evolución pormenorizada por sector, controlando por la dedicación del individuo la labor (véase el Anexo).

Figura 6. Ponderación del ingreso mensual y horario respecto a total SIC

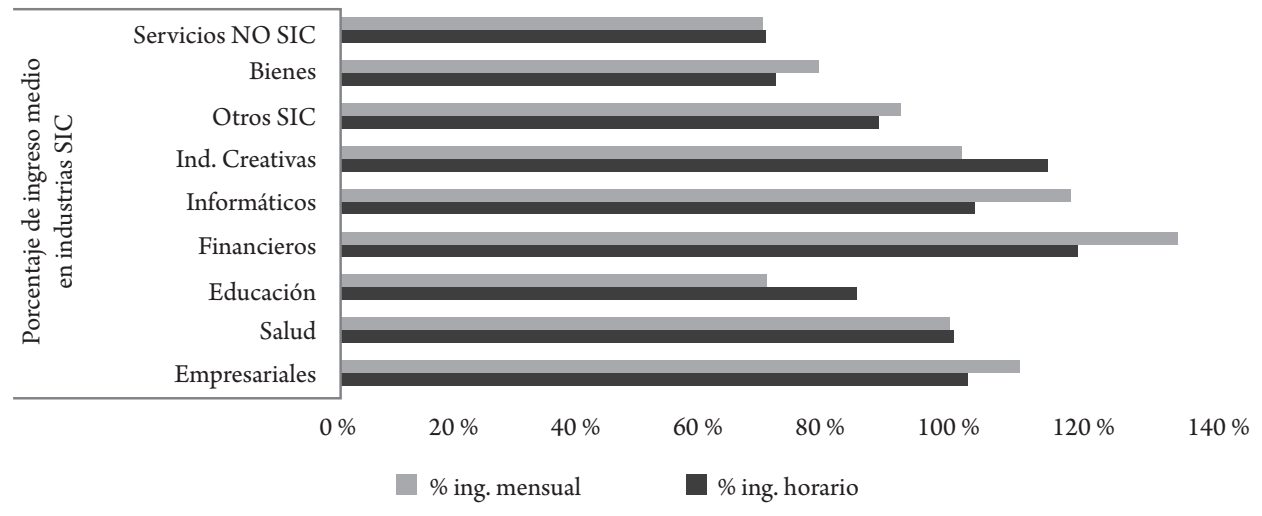

Fuente. Elaboración propia con base en datos de EAH de la DGEyC de CABA. 


\section{Discusión de resultados econométricos ${ }^{17}$}

A lo largo de este trabajo se analizó el rol del sector también sus diferencias frente al resto de los actores. Sin embargo, a pesar de obtener un buen mapa descriptivo, al analizarse de manera escindida se impide entender el genuino potencial explicativo de cada una de las variables.

Tabla 2. Coeficientes de regresión logística para sector SIC — expresados en ratio de odds_- ${ }^{18}$

\begin{tabular}{|c|c|c|c|c|c|c|c|c|}
\hline \multirow[b]{2}{*}{ Variables } & \multicolumn{2}{|c|}{ SIC } & \multicolumn{2}{|c|}{ SICs/ed } & \multicolumn{2}{|c|}{ SICCOGN } & \multicolumn{2}{|c|}{ SICPROF } \\
\hline & 2015 & 2018 & 2015 & 2018 & 2015 & 2018 & 2015 & 2018 \\
\hline \multirow[t]{2}{*}{ Genero } & $1,195372^{* * *}$ & $1,322329^{* * *}$ & $0,829204^{* * *}$ & $0,941113^{* * *}$ & $1,230135^{* * *}$ & $1,191822^{* * *}$ & $0,816743^{* * *}$ & $0,768417^{* * *}$ \\
\hline & $-0,004381-$ & $-0,004832-$ & $-0,003142-$ & $-0,003531-$ & $-0,005390-$ & $-0,005069-$ & $-0,004647-$ & $-0,004117-$ \\
\hline \multirow[t]{2}{*}{ Joven } & $0,951666^{* * *}$ & $0,912688^{* * *}$ & $1,049628^{* * *}$ & $1,042753^{* * *}$ & $0,851265^{* * *}$ & $0,882735^{* * *}$ & 0,990498 & $1,057564^{* * *}$ \\
\hline & $-0,004056-$ & $-0,003853$ - & $-0,004598-$ & $-0,004516-$ & $-0,004335-$ & $-0,004350-$ & $-0,006533-$ & $-0,006666-$ \\
\hline \multirow[t]{2}{*}{ Mayor } & $1,026089^{* * *}$ & $0,888714^{* * *}$ & 1,004147 & $0,966468^{* * *}$ & $1,041831^{* * *}$ & $0,949284^{* * *}$ & $1,150542^{* * *}$ & $1,436207^{* * *}$ \\
\hline & $-0,005060-$ & $-0,004332-$ & $-0,005103-$ & $-0,004814-$ & $-0,005973-$ & $-0,005268-$ & $-0,008503-$ & $-0,009770-$ \\
\hline \multirow[t]{2}{*}{ Educbaja } & $0,350194^{* * *}$ & $0,437533^{* * *}$ & $0,339047^{* * *}$ & $0,421858^{* * *}$ & $0,123892^{* * *}$ & $0,276018^{* * *}$ & $0,030688^{* * *}$ & $0,251996^{* * *}$ \\
\hline & $-0,002849-$ & $-0,003795-$ & $-0,003025-$ & $-0,004038-$ & $-0,002738-$ & $-0,005428-$ & $-0,002517-$ & $-0,009494-$ \\
\hline \multirow[t]{2}{*}{ Univinc } & $2,383933^{* * *}$ & $2,686571^{* * *}$ & $2,133384^{* * *}$ & $2,417264^{* * *}$ & $3,447278^{* * *}$ & $4,732681^{* * *}$ & $3,573519^{* * *}$ & $4,766973^{* * *}$ \\
\hline & $-0,013407-$ & $-0,016184-$ & $-0,012739-$ & $-0,015556-$ & $-0,029531-$ & $-0,047165-$ & $-0,055232-$ & $-0,084481-$ \\
\hline \multirow[t]{2}{*}{ Univcomp } & $4,756189^{* * *}$ & $5,655434^{* * *}$ & $3,781960^{* * *}$ & $4,499303^{* * *}$ & $8,662158^{* * *}$ & $12,515672^{* * *}$ & $19,683734^{* * *}$ & $22,104234^{* * *}$ \\
\hline & $-0,025098-$ & $-0,032229-$ & $-0,021039-$ & $-0,027222-$ & $-0,069072-$ & $-0,118798-$ & $-0,278646-$ & $-0,370847-$ \\
\hline \multirow[t]{2}{*}{ Migrante } & $0,510807^{* * *}$ & $0,491692^{* * *}$ & $0,639803^{* * *}$ & $0,610081^{* * *}$ & $0,617481^{* * *}$ & $0,702352^{* * *}$ & $1,070520^{* * *}$ & $0,740717^{* * *}$ \\
\hline & $-0,003110-$ & $-0,002645-$ & $-0,004023-$ & $-0,003395-$ & $-0,005014-$ & $-0,004559-$ & $-0,010657-$ & $-0,006111-$ \\
\hline \multirow[t]{2}{*}{ Sur } & $0,806778^{* * *}$ & $0,745341^{* * *}$ & $0,735215^{* * *}$ & $0,631105^{* * *}$ & $0,875087^{* * *}$ & $0,767145^{* * *}$ & $0.424797^{* * *}$ & $0,422830^{* * *}$ \\
\hline & $-0,004571-$ & $-0,004189-$ & $-0,004310-$ & $-0,003692-$ & $-0,006151-$ & $-0,005189-$ & $-0,004242-$ & $-0,004011-$ \\
\hline \multirow[t]{2}{*}{ Centro-Este } & $0,933957^{* * *}$ & $0,874724^{* * *}$ & $0,866035^{* * *}$ & $0,817610^{* * *}$ & $0,975070^{* * *}$ & $0,792848^{* * *}$ & $0,705922^{* * *}$ & $0,662535^{* * *}$ \\
\hline & $-0,004188-$ & $-0,003915-$ & $-0,003933-$ & $-0,003684-$ & $-0,005068-$ & $-0,004004-$ & $-0,004515-$ & $-0,004033-$ \\
\hline \multirow[t]{2}{*}{ Oeste } & $0,792878^{* * *}$ & $0,794471^{* * *}$ & $0,648200^{* * *}$ & $0,675683^{* * *}$ & $0,963200^{* * *}$ & $0,903037^{* * *}$ & $0.401956^{* * *}$ & $0,477176^{* * *}$ \\
\hline & $-0,004424-$ & $-0,004468-$ & $-0,003789-$ & $-0,003906-$ & $-0,006374-$ & $-0,005782-$ & $-0,003894-$ & $-0,004104-$ \\
\hline \multirow[t]{2}{*}{ Constant } & $0,299888^{* * *}$ & $0,271878^{* * *}$ & $0,285937^{* * *}$ & $0,248284^{* * *}$ & $0,070592^{* * *}$ & $0,058929^{* * *}$ & $0,031306^{* * *}$ & $0,026826^{* * *}$ \\
\hline & $-0,001862-$ & $-0,001820-$ & $-0,001844-$ & $-0,001740-$ & $-0,000636-$ & $-0,000611-$ & $-0,000470-$ & $-0,000470-$ \\
\hline Observations & $1,584,031$ & $1,585,655$ & $1,584,031$ & $1,585,655$ & $1,584,031$ & $1,585,655$ & $1,384,076$ & $1,395,974$ \\
\hline \multicolumn{9}{|c|}{ seEform in parentheses } \\
\hline${ }^{* * *} \mathrm{p}<0,01,{ }^{* *}$ & $0,05,{ }^{*} \mathrm{p}<0,1$ & & & & & & & \\
\hline
\end{tabular}

Fuente. elaboración propia con base en datos de EAH de la DGEyC de CABA.

\footnotetext{
${ }^{17}$ Es importante tener en cuenta que la regresión logística puede manifestar ciertas fuentes de endogeneidad en las variables. Estas posiblemente se pueden hallar entre las variables explicativas vinculadas a los niveles de educación y las relacionadas con el ingreso a un puesto de trabajo SIC. Es necesario destacar estas fuentes de endogeneidad, pero la solución econométrica a dichos problemas excede el marco de estudio de este trabajo.

${ }^{18}$ La regresión logística realizada cumple con los controles de autocorrelación y los controles de normalidad efectuados mediante el test de Jarque-Bera.
} 
En las regresiones planteadas se añade un primer control de género que obtiene el valor 1 para el sexo femenino. En ambos años se observa idéntica tendencia - aunque con diferencias menores en los valores - en la participación de las mujeres dentro de las industrias SIC. Si bien se demuestran mayores probabilidades de desempeño en el rubro - llegando al $32 \%$ adicional para el 2018 -, el efecto se revierte al excluir educación. Nótese que el potencial explicativo continúa siendo alto controlando por tipo de tareas, si se asume que las mujeres tienen una participación mucho más alta en tareas cognitivas en detrimento de las manuales.

Por su parte, al analizar tareas profesionales — de mayor remuneración - las mujeres presentan una relación negativa, ya que pueden entrever problemas de equidad vertical o «techo de cristal». Estos resultados encuentran cierto paralelismo en los resultados encontrados por el Gender Gap (World Economic Forum, 2018), en los cuales se señala que el rol de las mujeres en trabajos ligados a inteligencia artificial como proxy de una demanda de muy alta calificación observaban una amplia brecha, incluso a pesar de la innovación tecnológica y la caída en la demanda de tareas ligadas a la fuerza física.

Por otra parte, se incluyen dos variables dummy que tienen a un individuo de entre 36 y 50 años de referencia y que se fijan en el segmento Joven entre 18 y 35- y Mayor —entre 51 y 65 añosconsiderando solo aquella población activa de entre 18 y 65 años. Los jóvenes enuncian menores probabilidades de incorporarse a trabajos cognitivos de ingreso horario alto y menores chances de ubicarse en una tarea profesional respecto a las cohortes más altas, hecho estilizado que también se comprueba en naciones centrales (Bussolo, Davalos, Peragine y Sundaram, 2019).

La educación resulta el componente de mayor significatividad para explicar la posibilidad de ingreso a un empleo SIC, destacándose esta industria como la principal demandante de trabajo calificado. López et al. (2017) destacan la calificación de la mano de obra como uno de las principales determinantes para la competitividad de las empresas latinoamericanas exportadoras de servicios. Lennon (2008) alcanza una conclusión similar para una muestra más amplia de países y señala un claro impacto de la dotación educativa en la balanza comercial.

De forma esperable, el parámetro de Educbaja -aquellas calificaciones inferiores al secundario completo- se vuelve paulatinamente más alto en términos negativos dada la correlación inversaconforme aumentan las exigencias en el puesto. En efecto, los trabajos intensivos en tareas cognitivas no rutinarias exigen una calificación elevada, relegando las chances de individuos con una instrucción menor.

Por su parte, Univinc muestra cómo el paso por una institución terciaria, a pesar de la no cumplimentación, expande de manera significativa las posibilidades de arribo a las labores mencionadas - con una tendencia creciente en el tiempo-, lo cual rompe con las teorías credencialistas clásicas. Univcomp presenta lógicamente el mayor potencial explicativo, en línea con la importancia que el capital humano representa para este tipo de industrias, conforme lo expresado en capítulos anteriores. Del mismo modo, Apella y Zunino (2017) encontraban el aumento de la participación en tareas cognitivas fuertemente condicionado por el nivel de calificación y las habilidades que tienen los trabajadores.

La correlación entre educación superior y tareas de calificación profesional podría resultar obvia. Sin embargo, las credenciales educativas oferta de trabajo- no necesariamente resultan intercambiables por niveles de calificación en la tarea ni en los requerimientos del sector empleador. Groisman y Marshall (2013) señalan la ausencia de correspondencia para el caso argentino, verificando similares tareas realizadas desarrolladas por individuos con distinta dotación educativa, hecho que no parecería comprobarse en el sector SIC.

Usualmente se asimila al migrante con una menor dotación de capital, ya sea en términos físicos o de capital humano. Sin embargo, aun si se controla por distintos atributos individuales, observa la variable migrante con una considerable menor probabilidad 
de posicionamiento tanto a nivel ocupación como tarea. Estos resultados son coincidentes con el enfoque de Ottaviano y Peri (2012), quienes entienden a nativos y migrantes dentro de una misma «célula de habilidad»y no como sustitutos perfectos. Una potencial explicación puede deberse a la falta de estatus legal presente en muchos casos en los migrantes, lo cual los condiciona a tareas de menor remuneración, menor crecimiento salarial y menor retorno a la educación (Amuedo-Dorantes, Malo y Muñoz-Bullón, 2013).

Respecto a la interpretación de los controles geográficos, debemos añadir ciertos recaudos. Utilizando como valor de referencia a la zona Norte, tanto Centro-Este como Oeste y Sur presentan para quienes en ellas residan menores probabilidades de alcanzar estas posiciones laborales. Es aún más extremo para la región Sur —coincidente con lo señalado en secciones anteriores-, pues la correlación no induce el sentido de la causalidad.

Podría pensarse en un efecto de networking por el cual aquellos individuos que viven en regiones más acaudaladas tienen una mayor red de contactos que les permite escalar a las mejores posiciones laborales. Esto se encuentra en línea con estudios de desigualdad inducidos por externalidades de network y efectos de contactos (Di Maggio y Garip, 2012). Sin embargo, también puede pensarse de forma inversa: aquellos que desempeñan tareas mejor remuneradas deciden radicarse en regiones de mayor poder adquisitivo, dándose en este caso la causalidad desde el empleo hacia la región.

\section{Conclusiones}

as industrias SIC representan una parte
esencial del esquema laboral y productivo de la Ciudad Autónoma de Buenos Aires. Tanto por su contribución al crecimiento como su absorción de mano de obra se destaca en una región con una particular incidencia del sector servicios. Con una participación casi del $40 \%$ de la ocupación total, los servicios intensivos en conocimiento han potenciado una generación de trabajo que permite retornos muy por encima del promedio de los restantes sectores. Sin embargo, en el interior de la industria la disparidad en el salario tanto mensual como horario no dejó de ser elevada, también con notables diferencias en la absorción de empleo.

Además de las variables nodales - precio y cantidad-, el sector presentó un patrón de comportamiento signado por la radicación geográfica en el interior de la ciudad. Con una especial participación de la zona Norte en todos los sectores, la zona Sur aparece como la menos alcanzada por las bondades de la industria, incluso al controlar por las habituales características que definen la oferta laboral. Líneas de investigación posteriores merecen un análisis de la industria en regiones de menores recursos. Al extender la muestra al total país, es esperable que su alcance se vea considerablemente diluido.

Por su parte, las industrias SIC contienen una más alta participación en la generación de empleo, lo cual resulta complementario con el avance tecnológico e informático. Una vasta sección de la literatura muestra cómo aquellas ocupaciones que comprenden esencialmente tareas cognitivas no rutinarias son las que mayores aumentos de productividad exhiben frente a la interrelación con la robótica, la informática y otras herramientas tecnológicas. Por el contrario, y a diferencia del sector servicios No SIC y bienes, la ponderación en tareas rutinarias y manuales no rutinarias son considerablemente menores. Las primeras se enfrentan a un especial riesgo de automatización, mientras que las segundas plantean una remuneración que ha tendido no solo a la baja, sino también a posicionarse en la cola más baja de la distribución. 
Del mismo modo, las industrias SIC permiten una mayor generación de tareas profesionales - y técnicas en casos como servicios informáticos y educativos-, en línea con los efectos de spin off observados entre calificaciones altas y el uso de la tecnología. No es llamativo entonces que las industrias con mayor carga de empleo profesional sean las que mayores tareas cognitivas no rutinarias generen, lo que se traduce en remuneraciones muy por encima de la media y una productividad creciente.

Las posibilidades para un individuo de desempeñarse en alguno de los sectores que componen la industria SIC se encuentran íntimamente ligadas al capital humano general adquirido. Los estudios superiores, incluso a pesar de su no cumplimentación, tienen un rol protagónico en este proceso. Si bien el control por género parece en un principio privilegiar a las mujeres, al aislar los servicios educativos — tradicionalmente asociados a un rol femenino- las conclusiones parecen revertirse, al igual que lo hacen al poner el foco en la especificidad de las tareas. En efecto, problemas de equidad vertical parecen aflorar dadas las menores chances de alcanzar las «mejores» tareas dentro del rubro. Por último, la condición de migrante también conlleva cierta penalidad tanto en el arribo al sector como en las tareas desarrolladas en este.

A pesar de su escisión por rubros, este documento tuvo por objeto un análisis integral del sector SIC. El énfasis puesto en el plano laboral deja las puertas abiertas a un diseño específico por rama, cuyos requerimientos resultan tan disímiles como las habilidades de su fuerza de trabajo. Para esto, el aprovechamiento de nuevas y más complejas fuentes de datos aparece como otro desafío en paralelo que permita lograr un entendimiento más acabado del sector.

\section{Referencias}

Acemoglu, D.; Autor, D. (2011). Skills, tasks and technologies: implications for employment and earnings. En D. Card; O. Ashenfelter (Eds.) Handbook of labor economics. (1043-1171). Boston: North Holland. DOI: https://doi.org/10.1016/ S0169-7218(11)02410-5

Acemoglu, D.; Restrepo, P. (2017a). Secular stagnation? The effect of aging on economic growth in the age of automation. American Economic Review, 107(5), 79-174. DOI: https://doi. org/10.1257/aer.p20171101

Acemoglu, D.; Restrepo, P. (Marzo de 2017b). Robots and jobs. National Bureau of Economic Research, Working paper 23285, Cambridge, EE.UU. DOI: https://doi.org/10.3386/w23285

Acemoglu, D.; Restrepo, P. (Enero de 2018). Artificial intelligence, automation and work. National Bureau of Economic Research, Working paper 24196. Cambridge, EE.UU. DOI: https://doi. org/10.3386/w24196

Ameudo-Dorantes, C.; Malo, M. A.; Muñoz-Bullón, F. (2013). New evidence on the impact of legal status on immigrant labor market performance: the Spanish case. Labour, 27(1), 93-113. DOI: https://doi.org/10.1111/labr.12002

Apella, I. R.; Zunino, G. (2017). Cambio tecnológico y mercado de trabajo en Argentina y Uruguay. Un análisis desde el enfoque de tareas. Montevideo: Oficina del Banco Mundial.
Atkinson, A. B. (2008). The Changing Distribution of Earnings in OECD Countries. New York: Oxford University Press. DOI: https://doi.org/10.1093/ acprof:oso/9780199532438.001.0001

Autor, D. H.; Dorn, D. (24 de agosto de 2013). How technology wrecks the middle class. The New York Times. Recuperado de https://nyti.ms/37zEJ91

Autor, D.H.; Handel, M.J. (2013). Putting tasks to the test: human capital, job tasks, and wages. Journal of Labor Economics, 31(2), S59-S96. DOI: https://doi.org/10.1086/669332

Autor, D. H.; Katz, L. F.; Krueger, A. B. (1998). Computing inequality: have computers changed the labor market? The Quarterly Journal of Economics, 113(4), 1169-1213. DOI: https://doi.org/10.1162/003355398555874

Autor, D. H.; Levy, F.; Murnane, R. J. (2003). The skill content of recent technological change: an empirical exploration. The Quarterly Journal of Economics, 118(4), 1279-1333. DOI: https://doi.org/10.1162/003355303322552801

Banco Mundial. (2016). Informe sobre el desarrollo mundial 2016: dividendos digitales. Washington D. C.: Banco Mundial.

Bresnahan, T. F. (1999). Computerisation and wage dispersion: an analytical reinterpretation. The Economic Journal, 109(456), 390-415. DOI: https://doi.org/10.1111/1468-0297.00442 
Bresnahan, T. F.; Brynjolfsson, E.; Hitt, L. M. (2002). Information technology, workplace organization, and the demand for skilled labor: firm-level evidence. The Quarterly Journal of Economics, 117(1), 339-376. DOI: https://doi. org/10.1162/0033553027533399526

Bussolo, M.; Davalos, M. E.; Peragine, V.; Sundaram, R. (2019). Toward a new social contract: taking on distributional tensions in Europe and Central Asia. Washington D.C.: International Bank for Reconstruction and Development, The World Bank.

Dauth, W.; Findeisen, S.; Südekum, J.; Wößner, N. (2017). German robots. The impact on workers. Londres: Centre for Economic Policy Research

DiMaggio, P.; Garip, F. (2012). Network effects and social inequality. Annual Review of Sociology, 38, 93-118. DOI: https://doi.org/10.1146/annurev.soc.012809.102545

Escaith, H. (2008). Measuring trade in value added in the new industrial economy: statistical implications. 12th Symposium French National Account Association. París, 4-6 de junio. DOI: https://doi.org/10.2139/ssrn.1189162

Frey, C. B.; Osborne, M. A. (2013). The future of employment: how susceptible are jobs to computerisation? Technological Forecasting and Social Change, 114, 254-280. DOI: https:// doi.org/10.1016/j.techfore.2016.08.019

Guerrieri, P.; Meliciani, V. (2009). Internationalization, Technology and Business Services Specialization in Europe. Ponencia presentada en Italy's External Competitiveness. Roma, 24 y 25 de noviembre.

Goldin, C.; Katz, L. F. (2007). The race between education and technology: the evolution of U. S. educational wage differentials, 1890 to 2005. En NBER (Ed.) The Intimate Contest between Education and Technology (N.o w12984). Cambridge: National Bureau of Economic Research. DOI: https://doi.org/10.3386/w12984

Groisman, F. A.; Marshall, A. (2013). Educación, demanda de calificaciones y salarios relativos: el caso argentino, 20042011. Desarrollo Económico, 55(216), 227-243.

Lennon, C. (2008). Trade in services and trade in goods: differences and complementarities. París, Francia. Recuperado de https:// bit.ly/2VLIJy5

López, A.; Ramos, D. (2013). ¿Pueden los servicios intensivos en conocimiento ser un nuevo motor de crecimiento en América Latina? Revista Iberoamericana de Ciencia, Tecnología $y$ Sociedad, 8(24), 83-115.
López, A.; Niembro, A.; Ramos, D. (2017). Service companies in Latin America: an explanatory study about determinants of international competitiveness, obstacles and public policies. Tec Empresarial, 11(1), 7-22. DOI: https://doi. org/10.18845/te.v11i1.3099

Martínez, R. G.; Lo Cascio, J.; Leone, J. (2018). Brechas socioeconómicas al interior de CABA. Una propuesta desde la medición multidimensional de la pobreza. Cuadernos de investigación. Serie Económica, (7), 70-93.

Meyer, T. (2007). India's specialisation in IT exports: offshoring can't defy gravity. Research Notes, (27), 1-24.

Miles, I.; Kastrinos, N.; Flanagan, K.; Bilderbeek, R.; Den Hertog, P.; Huntink, W.; Bouman, M. (1995). Knowledge-intensive business services: users, carriers and sources of innovation. EIMS Publication 15. Amsterdam: Nederlands Instituut voor Arbeidsomstandigheden.

Murphy, K. M.; Riddell, W. C.; Romer, P. M. (1998). Wages, Skills, and Technology in the United States and Canada. NBER Working Papers 6638. Massachusetts: National Bureau of Economic Research, Inc. DOI: https://doi.org/10.3386/w6638

Nyahoho, E. (2010). Determinants of comparative advantage in the international trade of services: an empirical study of the Hecksher-Ohlin Approach. Global Economy Journal, 10(1), 1850186. DOI: https://doi.org/10.2202/1524-5861.1495

OECD. (2013). Interconected economies: benefiting from global value chains. OECD Publishing.

Picketty, T.; Saenz, E. (2014). Inequality in the long run. Science, 334, 838-843. DOI: https://doi.org/10.1126/ science. 1251936

Ottaviano, G.; Peri, G. (2012). Rethinking the effect of inmigration on wages. Journal of the European Economic Association, 10(1), 152-197. DOI: https://doi.org/10.1111/ j.1542-4774.2011.01052.x

World Economic Forum. (2018). The Global Gender Gap Report. Ginebra: World Economic Forum. 


\section{Anexo}

Tabla A1. Contribución al crecimiento del PBG de CABA en las fases cíclicas, en porcentajes

\begin{tabular}{|c|c|c|c|c|c|}
\hline \multirow[t]{2}{*}{ Categoría } & \multicolumn{5}{|c|}{ Fases cíclicas del PBG de CABA } \\
\hline & $\begin{array}{c}1995- \\
1998\end{array}$ & $\begin{array}{l}1998- \\
2002\end{array}$ & $\begin{array}{l}2002- \\
2009\end{array}$ & $\begin{array}{l}2009- \\
2014\end{array}$ & $\begin{array}{l}2014- \\
2017\end{array}$ \\
\hline Producto bruto geográfico & 100,0 & 100,0 & 100,0 & 100,0 & 100,0 \\
\hline SIC & 51,3 & 23,0 & 33,7 & 45,1 & 52,4 \\
\hline Servicios empresariales & 19,3 & 13,5 & 17,2 & 11,6 & 25,2 \\
\hline Servicios sociales y de salud & 1,6 & $-1,0$ & 10,1 & 15,0 & 22,5 \\
\hline Enseñanza & 1,1 & $-0,2$ & 5,0 & 0,9 & 4,6 \\
\hline Intermediación financiera y otros servicios financieros & 29,2 & 10,7 & 1,4 & 17,7 & 0,1 \\
\hline NO SIC & 48,7 & 77,0 & 66,3 & 54,9 & 47,6 \\
\hline Agricultura, ganadería, pesca y minas y canteras & $-1,0$ & $-0,9$ & 3,0 & 1,8 & $-3,4$ \\
\hline Industria manufacturera & 15,2 & 27,8 & 5,5 & 10,3 & $-10,6$ \\
\hline Electricidad, gas y agua & $-0,6$ & $-0,1$ & 2,3 & $-0,1$ & 1,0 \\
\hline Construcción & 2,8 & 7,8 & 13,3 & $-0,2$ & 13,1 \\
\hline Comercio & 23,7 & 34,4 & 1,8 & 20,7 & $-4,0$ \\
\hline Servicios de hotelería y restaurantes & 4,0 & 1,6 & 6,4 & 2,0 & 14,4 \\
\hline Transporte, almacenamiento y comunicaciones & 4,6 & 5,2 & 18,5 & 9,6 & 33,1 \\
\hline Administración pública, defensa y seguridad social & $-3,3$ & 1,5 & 4,5 & 7,7 & 3,7 \\
\hline Servicios comunitarios, sociales y personales n. c. p. & 2,9 & $-0,5$ & 9,8 & 3,4 & 2,5 \\
\hline Servicio doméstico & 0,5 & 0,0 & 1,3 & $-0,4$ & $-2,3$ \\
\hline
\end{tabular}

Fuente. Elaboración propia con base en datos de la Dirección de Estadística y Censos de la Gobernación de la Ciudad de Buenos Aires. 
Tabla A2. Salario horario por comuna y total CABA para cada actividad, en los años 2013, 2015 y 2018

\begin{tabular}{l|ccc|ccc|ccc}
\multicolumn{3}{c}{} & \multicolumn{3}{c}{ Total SIC } & \multicolumn{3}{c}{ Bienes } \\
& $\mathbf{2 0 1 3}$ & $\mathbf{2 0 1 5}$ & $\mathbf{2 0 1 8}$ & $\mathbf{2 0 1 3}$ & $\mathbf{2 0 1 5}$ & $\mathbf{2 0 1 8}$ & $\mathbf{2 0 1 3}$ & $\mathbf{2 0 1 5}$ & $\mathbf{2 0 1 8}$ \\
CABA & 269,76 & 222,70 & 211,97 & 189,17 & 159,60 & 190,01 & 178,35 & 155,62 & 143,91 \\
Norte & 300,80 & 228,64 & 243,47 & 278,07 & 211,23 & 268,82 & 220,10 & 199,07 & 174,64 \\
Cnt.-Este & 259,79 & 171,53 & 211,13 & 189,48 & 157,02 & 154,60 & 173,32 & 147,32 & 138,47 \\
Oeste & 231,64 & 164,64 & 181,36 & 167,69 & 145,45 & 140,77 & 181,55 & 144,19 & 145,32 \\
Sur & 251,63 & 143,23 & 165,07 & 116,33 & 119,24 & 111,46 & 133,12 & 130,67 & 120,06
\end{tabular}

\begin{tabular}{l|lll|lll|lll|l|lll}
\multicolumn{4}{c}{} & \multicolumn{3}{c}{ Serv. Empresariales } & \multicolumn{3}{c}{ Servicios de salud } & \multicolumn{3}{c}{ Servicios de educación } & \multicolumn{2}{c}{ Servicios financieros } \\
& $\mathbf{2 0 1 3}$ & $\mathbf{2 0 1 5}$ & $\mathbf{2 0 1 8}$ & $\mathbf{2 0 1 3}$ & $\mathbf{2 0 1 5}$ & $\mathbf{2 0 1 8}$ & $\mathbf{2 0 1 3}$ & $\mathbf{2 0 1 5}$ & $\mathbf{2 0 1 8}$ & $\mathbf{2 0 1 3}$ & $\mathbf{2 0 1 5}$ & $\mathbf{2 0 1 8}$ \\
CABA & 297,9 & 230,6 & 239,2 & 252,3 & 225,1 & 190,0 & 332,5 & 189,6 & 179,1 & 217,6 & 271,1 & 267,6 \\
Norte & 373,8 & 248,2 & 267,2 & 252,2 & 261,6 & 237,8 & 330,1 & 204,0 & 188,2 & 241,5 & 349,1 & 266,6 \\
Cnt.-Este & 251,3 & 213,5 & 229,8 & 249,3 & 209,1 & 173,4 & 351,2 & 192,7 & 196,4 & 218,9 & 242,9 & 319,9 \\
Oeste & 241,5 & 233,0 & 210,8 & 271,0 & 244,9 & 178,5 & 243,3 & 170,1 & 160,3 & 186,4 & 226,8 & 230,8 \\
Sur & 196,0 & 189,7 & 172,6 & 241,6 & 159,8 & 142,8 & 417,3 & 179,5 & 151,5 & 178,6 & 232,9 & 186,4
\end{tabular}

\begin{tabular}{|c|c|c|c|c|c|c|c|c|c|}
\hline & \multicolumn{3}{|c|}{ Servicios informáticos } & \multicolumn{3}{|c|}{ Industrias creativas } & \multicolumn{3}{|c|}{ Otros SIC } \\
\hline & 2013 & 2015 & 2018 & 2013 & 2015 & 2018 & 2013 & 2015 & 2018 \\
\hline CABA & 226,1 & 233,2 & 248,5 & 301,0 & 259,7 & 200,5 & 190,9 & 197,2 & 189,6 \\
\hline Norte & 253,6 & 241,5 & 299,0 & 345,2 & 322,4 & 203,0 & 215,6 & 244,1 & 245,2 \\
\hline $\begin{array}{l}\text { Cnt.-Es- } \\
\text { te }\end{array}$ & 205,2 & 210,9 & 197,9 & 275,3 & 241,1 & 222,4 & 183,1 & 177,9 & 169,6 \\
\hline Oeste & 204,0 & 276,4 & 161,9 & 228,1 & 152,3 & 162,3 & 177,3 & 174,9 & 164,0 \\
\hline Sur & 180,5 & 244,9 & 282,0 & 262,7 & 150,7 & 158,8 & 169,5 & 179,8 & 159,1 \\
\hline
\end{tabular}

Fuente. Elaboración propia con base en datos de EAH de la DGEyC de CABA. 